\title{
New methylphenylsiloxane resin based on alkoxysilanes
}

\author{
(C) Andrey M. Kontorov
}

State Research Center Joint Stock Company "State Scientific-Research Institute of Chemistry and Technology of Organoelement Compounds”. Entuziastov Ave., 38. Moscow, 111123. Russia. E-mail: ankont2@yandex.ru

Keywords: methylphenylsiloxane resin, oligosilsesquioxanes, thermal and thermo-oxidative stability, spectroscopy ${ }^{29} \mathrm{Si},{ }^{1} \mathrm{~N}$ NMR.

\section{Abstract}

Currently interested in the reaction for producing oligosilsesquioxanes based on acidolysis alkoxysilanes. In this regard, the author was given the following tasks: development of optimal synthesis conditions; preparation of new methylphenylsiloxane resins with different properties.

In this paper, the properties of new methylphenylsiloxane resins (MPR) with various radicals in silicon were studied. New IFSS were obtained by a new universal technology-acidolysis of a mixture of methyltriethoxysilane (MTEOS) and phenyltriethoxysilane (PTEOS) with various radicals, which are environmentally friendly raw materials. The obtained MPR were characterized by NMR spectroscopy on ${ }^{1} \mathrm{H}$ and ${ }^{29} \mathrm{Si}$ nuclei. Spectra were recorded at room temperature in deuteroacetone using Bruker AM-360 Fourier spectrometer. ${ }^{29} \mathrm{Si}$ NMR spectra were measured using the pulse program "Inverse Gated Heteronuclear Decoupling".

The content of residual functional groups (Si-OH, Si-OEt) in IFSS was determined by functional analysis methods. Determination of ethoxy groups and hydroxy groups was carried out by iodometric and aluminohydride method, respectively.

Thermogravimetric analysis was performed on the device Derivatograph-H (firm Mom). TGA studies were carried out in the argon atmosphere and in the air at a heating rate of $10^{\circ} \mathrm{C} / \mathrm{min}$.

Measurements of kinematic viscosity of $20 \%$ and $50 \%$ by weight. toluene solutions of MPR were carried out at $20^{\circ} \mathrm{C}$ on the viscometer HPV-2.

The reaction acidolysis of methyltriethoxysilane and oligophenylenes is a convenient and versatile method for the synthesis of new heat-resistant resins methylphenylsiloxanes. In the course of the study, it was found that the resins obtained on the basis of organoalkoxysilanes are characterized by higher thermal and thermo-oxidative stability.

\section{References}

[1] L.M. Khananashvili, K.A. Andrianov. Technology of Organoelement monomers and polymers. Moscow: Chemistry. 1983. 400p. (russian)

[2] M.V. Sobolevsky, O.A. Muzovskaya, G.S. Popelova. Properties and applications of silicone products. Moscow: Chemistry. 1975. 296p. (russian)

[3] O.G. Ryzhova, A.M. Kontorov, A.N. Polivanov, M.U. Buzin. New thermostable oligomethylphenylsiloxanes on the basis of organoalkoxysilanes different functionality. $5^{\text {th }}$ European organosilicon days. Vienna. 2009. P.183.

[4] E.V. Egorov, N.G. Vasilenko, N.V. Demchenko, E.A. Tatarinova, A.M. Muzafarov. Polycondensation of alkoxysilanes in an active medium is a universal method of obtaining polyorganosiloxanes. Reports of the Academy of Sciences, 2009. Vol.424. No.2. P.200-204. (russian)

[5] Method of producing polyorganosiloxanes based on organoalkoxysilanes. Patent of Russia № 2428438 S2, from 10.09.2011. O.G. Ryzhova, P.A. Storozhenko, A.N. Polivanov, A.M. Kontorov, G. Ya. Zhigalin, O.L. Yershov, B.E. Kozhevnikov, A.V. Apal'kov, M.N. Perveev. (russian)

[6] Method of producing heat-resistant resins oligoorganosiloxanes. Patent of Russia №2464286 Cl, from 20.10.2012. A.M. Kontorov, G.Ya. Zhigalin, O.L. Yershov. (russian)

[7] Enamel heat resistant. Patent of Russia № 2495895 C1, from 10.05.2012. A.I. Katanaev, R.S. Mirgazitova, A.N. Polivanov, O.G. Ryzhova, A.M. Kontorov. (russian)

[8] H.W. Ahn. Synthesis, characterisation and computer simulations of stereoregular poly (methylphenylsiloxane). PhD Thesis. 2003.

[9] D. Pinkert, C. Limberg. Iron Silicates, Iron-Modulated Zeolite Catalysts, and Molecular Models Thereof. European Journal. 2014. No.30. P.9167-9175. 
[10] P. Zak, B. Dudziec, M. Kubiecki, B. Marciniec. "Silylative Coupling versus Metathesis-Efficient Methods for synthesis of Difunctionalised Double-Decker Silsesquioxane Derivatives". European Journal. 2014. No.30. P.9387-9393.

[11] Electric wire covering material. Patent of China CN104059330. 2014. Zhang, Haoliang.

[12] Polysiloxane-modified thermoplastic material and its preparation method. Patent of China CN102924793. 2013. Li, Yanmin.

[13] Silane coupler-modified epoxy resin adhesive for optical fiber. Patent of China CN102766432. 2012. Cao, Xianglai; Kong, Qingru; Qiao, Yi.

[14] Polycarbonate resin composition with good flame retardancy and light stability and use for LCD backlight. Patent of United States US 20090239975. 2009. Jung, Hyuk Jin; Lim, Jong Cheol; Lee, Sang Hwa; Kim, Jong Yeu.

[15] Primer and finishing coating for high temperature exhaust pipe. Patent of China CN101531867. 2009. Zhu, Hongjiang.

[16] Organic silicone potting materials with high hot moisture and weather resistance for electronics. Patent of China CN101016446. 2007. Su, Junliu; Tang, Shengshan; Li, Jiazhong.

[17] Environment-friendly flameproof composite paints. Patent of China CN1995246. 2007. Yao, Qian; Yao, Xin; Yao, Huiwo.

[18] A.G. Ivanov, V.M. Kopylov, V.L. Ivanova, V.A. Kovyazin, I.B. Sokol'skaya, I.I. Khazanov. Getting organoalkoxysilanes partial acidolysis organoalkoxysilanes. Journal of General chemistry. 2012. Vol.82. Iss.1. P.69-75. (russian)

[19] Method of producing organosilicon resins. Patent of Russia № 2079516, from 25.06.1993. E.A. Chuprova and B.V. Molchanov. (russian) 